\title{
Simulation based Modeling and Implementation of Adaptive Control Technique for Non Linear Process Tank
}

\author{
P.Aravind \\ PG Scholar, \\ Department of Control and \\ Instrumentation Engineering, \\ JJ College of Engineering and \\ Technology,Trichirappalli-620 \\ 009, Tamil Nadu, India
}

\author{
M.Valluvan \\ Assistant Professor, \\ Department of Electronics and \\ Instrumentation Engineering, \\ JJ College of Engineering and \\ Technology, Trichirappalli-620 \\ 009, Tamil Nadu, India
}

\author{
M. Saranya \\ PG Scholar, \\ Department of Control and \\ Instrumentation Engineering, \\ $\mathrm{JJ}$ College of Engineering and \\ Technology,Trichirappalli-620 \\ 009, Tamil Nadu, India
}

\begin{abstract}
Control of nonlinear process is a complicated task in industrial environment. In this work, adaptive control technique is discussed in control of single conical tank level control system is a nonlinear system is identified mathematically. Analytical modeling were implemented and simulated in MATLAB SIMULINK and transfer function isobtain from the simulated response and PI controller parameter were derived for implementing gain scheduling adaptive controller and synthesis based method is used to obtain PI parameters for multiple linear models. The simulation studies were carried out for two controller parameters. From the results based on Performance indices like Integral Squared Error (ISE), it is proved the controller implemented using gain scheduling adaptive control technique out performs well over synthesis method based tuned multi PI controller.
\end{abstract}

\section{General Terms}

Process Control, Adaptive Control.

\section{Keywords}

Conical Tank, Synthesis Method, Gain Scheduling, MATLAB, Non Linear Process.

\section{INTRODUCTION}

In conical tank with gravity discharge flow are used as an inexpensive way to slurries and solids such as lime bark and coal to unit operation. The conical tank prevents the accumulation of solid at the bottom of the tank. Conical tanks are widely used in process industries. It gives a non-linearity because of its change in shape. Level response of conical tank varies with its volume geometry. The tuning of level controllers can be challenging because of the extreme variation in the process dynamics and tuning settings. Control system studies have shown that the most frequent method of optimum vales of controller parameters. Generally PI controller is suitable for to obtain a desired response.In particular, this exposes the fundamental modeling principle of fluid mass balance and a feedback control design methodology for a state-coupled, two-tank level control system.
The level control is a type of control method for common in process system. It must be controlled by the proper controller. The objective of the controller in the level control is to maintain a level at a given set point value and be able to accept new set point values dynamically. The conventional Proportional - Integral - Derivative (PID) is commonly utilized in controlling the level, but the parameter is not enough for efficient control. This paper endeavors to design a system using two methods of direct synthesis method and gain scheduling method of obtaining controller parameters In the Direct Synthesis (DS) method, the controller design is based on a process model and a desired closed-loop transfer function. The latter is usually specified for set-point changes, but responses to disturbances can also be utilized. Although these feedback controllers do not always have a PID structure, the DS method does produce PI or PID controllers for common process models. The second method is gain scheduling control improve the quality of control action. When deriving a simple model to be used for PID controller tuning, it is important to ensure that the model describes the process well for the typical input signal is obtained during the process operations model accuracy may be poor if the process is non-linear.

The process tank considered here has nonlinear characteristics which is represented as piecewise linearized models; multiple linear models of tank with many PI controllers were implemented. The proportional-integral (PI) and proportional integral- derivative (PID) controllers are widely used in many industrial control systems for several decades since Ziegler and Nichols [1] proposed their first PID tuning method. This is because the PID controller structure is simple and its principle is easier to understand than most other advanced controllers. Still much research has been going on in tuning the PID controllers for different process like large dead time, integrating process and First Order Process with Dead Time (FOPDT). Artificial Neural Network modeling and multivariable Model Predictive Controllers are designed by Rahul Shridhar, Douglas. J. Cooper [3] explained Ants colony optimization in level control of conical tank. A PI-type fuzzy controller that uses information from the fuzzy regions of a nonlinear process such as a continuously stirred tank reactor for $\mathrm{pH}$ titration is proposed by Qin et al. [4].Unconstrained 
multivariable Tuning was proposed by R. Shridhar, D. J. Cooper [5].Optimization algorithms are another area that has been receiving increased attention in the past few years by the research community as well as the industry [7].Swati Mohanty[8] designed Model Predictive Controller for flotation column. N.S. Bhuvaneswari , G. Uma , T.R. Rangaswamy carried out experiments in conical tank level control using Neural Network controllers [9].

\section{PROCESS DESCRIPTION}

A dynamic model of this process contains ordinary differential equations, which arise from the total mass balance on each of the tanks. We assume constant density. The process sketched in Figure.1 consists of vertical conical tank. The feed of water to the tank comes from an upstream unit. The liquid level in the tank is controlled by manipulating the flow rate of liquid pumped from the tank. The liquid level i.e. height $h$ is utilized for controller design. The height $h$ is maintained at desired level.

Fin

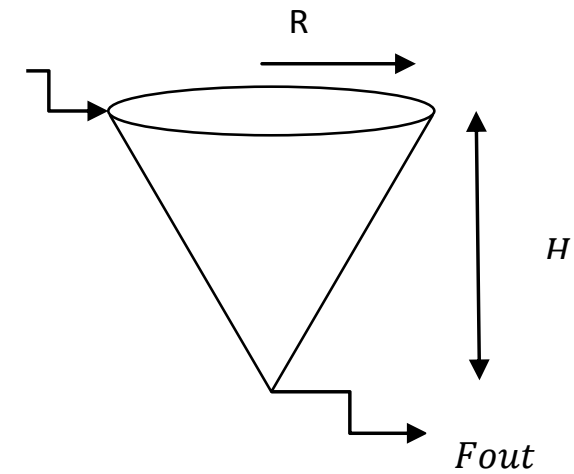

Figure 1: Schematic of Process Tank

\section{PROBLEM FORMULATION}

Table 1.Operating Parameters

\begin{tabular}{|c|c|c|c|}
\hline $\begin{array}{r}\text { Sl. } \\
\text { No }\end{array}$ & Parameter & Description & Value \\
\hline 1 & $\mathrm{R}$ & Total radius of the cone & $19.25 \mathrm{~cm}$ \\
\hline 2 & $\mathrm{H}$ & $\begin{array}{r}\text { Height of the tank } \\
\text { tank }\end{array}$ & $73 \mathrm{~cm}$ \\
\hline 3 & Fin & $\begin{array}{r}\text { Maximum inflow rate of the } \\
400 \mathrm{LPH}\end{array}$ & $55 \mathrm{~cm}{ }^{2} / \mathrm{s}$ \\
\hline 4 & $\beta$ & Value co-efficient & \multicolumn{2}{|c}{} \\
\hline
\end{tabular}

\subsection{Linearization}

In this paper, a linear model is obtained by transient response analysis of a nonlinear process tank [process variable height (h)]; this means a controller is tuned based on this model which works well only at this operating point. To obtain a linear model process steady state input - output characteristics curve is divided into four different linear regions as shown in the Figure 4.

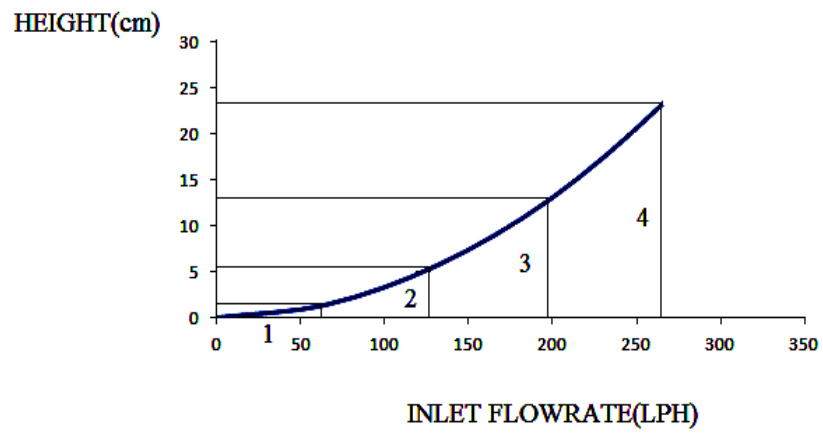

Figure 2: Piecewise Linearization of Input and Output Characteristics

\section{DESIGN OF CONTROLLER}

\subsection{Direct Synthesis Method}

Direct Synthesis is a model based tuning technique. It uses an identified process model in conjunction with a user specified closed loop response characteristic. This is a model based tuning technique. It uses an identified process model in conjunction with a user specified closed loop response characteristic. An advantage of this approach is that it provides insight into the role of the 'model' in control system design.

The overall transfer function for set point change assuming,

$\mathrm{G}_{\mathrm{m}}=\mathrm{G}_{\mathrm{v}}=1$

$\mathrm{G}_{\mathrm{sp}}=\frac{\mathrm{y}}{\mathrm{r}}=\frac{\mathrm{G}_{\mathrm{p}} \mathrm{G}_{\mathrm{c}}}{1+\mathrm{G}_{\mathrm{p}} \mathrm{G}_{\mathrm{c}}}$

On rearranging the equation (2), we get

$\mathrm{G}_{\mathrm{c}}=\frac{1}{\mathrm{G}_{\mathrm{p}}}\left[\frac{\mathrm{y} / \mathrm{r}}{1-\left(\frac{\mathrm{y}}{\mathrm{r}}\right)}\right]=\frac{1}{\mathrm{G}_{\mathrm{p}}}\left[\frac{\mathrm{G}_{\mathrm{sp}}}{1-\mathrm{G}_{\mathrm{sp}}}\right](3)$

Remarks on the direct synthesis method:

1. It depends heavily on the model type.

2. It requires model inversion, which may cause problem for non-minimum phase processes.

3. PID controller may not be realized unless an appropriate model form is used to synthesis the control law.

\subsection{Adaptive Control}

Research in adaptive control was started in the early 1950's in autopilot design for high-performance aircraft. Adaptive control deals with complex systems that have unpredictable parameter deviations and uncertainties. Its basic objective is to maintain consistent performance of a system in the presence of uncertainty and variations in plant parameters and adaptive control is superior to robust control in dealing with uncertainties in constant or slow-varying parameters. Robust 
control has advantages in dealing with disturbances, quickly varying parameters, and unmodeled dynamics. Adaptive controller is a dynamic system with on-line parameter estimation. Basic Ideas in Adaptive Control are estimation of uncertain plant / controller parameters on-line, while using measured system signals estimated parameters were used in control input computation.

\subsubsection{Gain Scheduling Method}

One of the most popular approaches to nonlinear control design divide and conquer type of design procedure nonlinear control design task is broken into a number of linear subproblems. It enables linear design methods to be applied to nonlinear problems'. It is considered 'open-loop adaptive control. In this method, a controller which improves the quality of control action, gain scheduling is a very effective way of controlling system whose dynamics changes with the operating condition. At each operating points controller parameters were obtained which relates to process parameters.

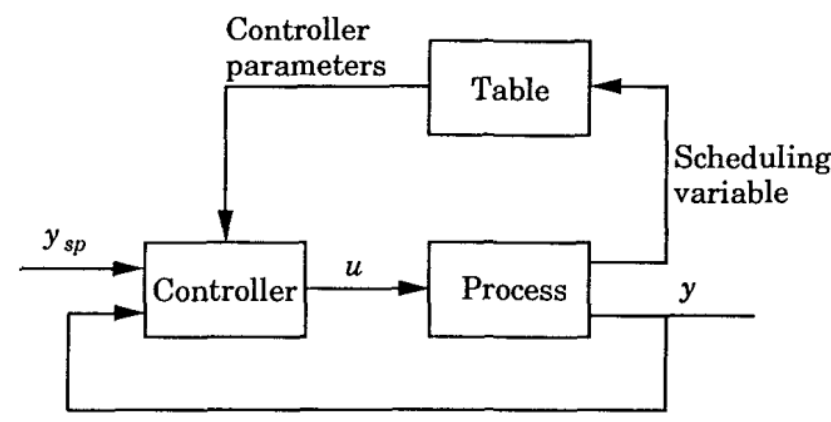

Figure3: Block diagram of a system with gain scheduling

\section{MODELING AND SIMULATION}

Observe that the nonlinear process model can be linearized around a particular steady-state value, and with this linearized approximation of the true nonlinear model, we can obtain fairly general results regarding the process behavior. The model which with sufficient accuracy describes physical processes of conical tank system. The theoretical model of various process units is derived by using the equations of mass balance and/or energy balance as follows:

Input - Output $=$ Rate of Accumulation

In a steady state condition, the accumulation is equal to zero. For dynamic simulation, the accumulation term to the mass and energy balance must be added. Feedback control systems are often referred to as closed-loop control systems. In a closed-loop control system the actuating error signal, which is the difference between the input signal and the feedback signal, is fed to the controller so as to reduce the error and bring the output of the system to a desired value.

The conical tank system, which exhibits the property of nonlinearity, mathematical model is obtained and simulated in SIMULINK. The process dynamics are analyzed in four segments so as to obtain effective models for the operating ranges.
The structure of single conical tank system is illustrated in Figure1. The tank level process to be simulated is single-input single-output (SISO) tank system. The user can adjust the inlet flow by adjusting the control signal, Fin. During the simulation, the level ' $h$ ' will be calculated at any instant of time. In the SISO tank system, the liquid will flow into the tank through inlet and the liquid will come out from the tank through outlet. Here, we want to maintain the level of the liquid in the tank at desired value; so the measured output variable is the liquid level $h$.

\subsection{Mathematical Modeling}

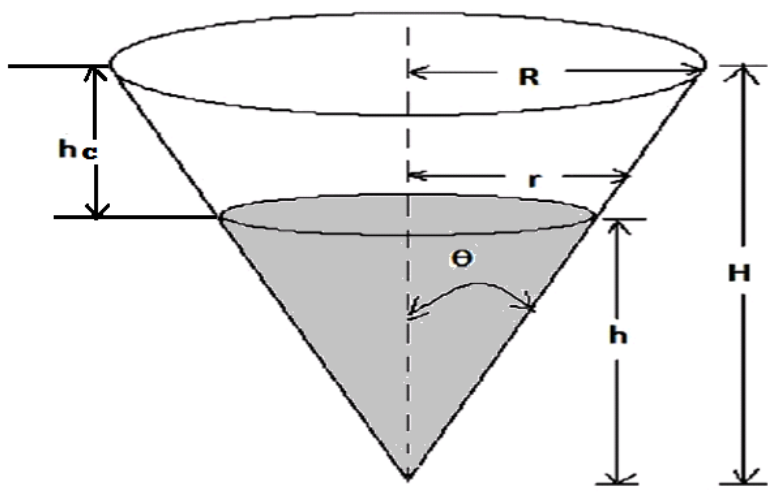

Figure 4: Mathematical Modeling for Conical Tank Where,

$\tan \theta=\frac{\mathrm{r}}{\mathrm{h}}=\frac{R}{H}$

$\mathrm{r}=\mathrm{R} * \frac{\mathrm{h}}{\mathrm{H}}$

A mathematical model is derived according to Law of conservation of mass for single conical tank,

Inflow rate - Outflow rate $=$ Accumulation

Fin - Fout $=\frac{\mathrm{d}}{\mathrm{dt}}[$ Total Volume of Conical Tank Conical Tank Cap Volume](6)

Total volume of the conical tank is $=\frac{1}{3} \pi r^{2} H$

Fin - Fout $=\frac{\mathrm{d}}{\mathrm{dt}}\left[\frac{1}{3} \pi r^{2} H-\frac{1}{3} \pi r^{2}\left(h_{c}\right)\right]$

Where hc is the cap height, $\mathrm{hc}=\mathrm{H}-\mathrm{h}$

Solving equation 7 we get,

$$
\begin{aligned}
& \text { Fin }- \text { Fout }=\frac{\mathrm{d}}{\mathrm{dt}}\left[\frac{1}{3} \pi r^{2} h\right] \\
& \frac{\mathrm{dh}}{\mathrm{dt}}=(\text { Fin }-\beta \sqrt{\mathrm{h}}) * \frac{1}{A}
\end{aligned}
$$


Where, $\mathrm{A}=\frac{1}{3} \pi r^{2} \mathrm{~h}$

The equation (9) describing the mathematical model for single conical tank level control, this equation is implemented in MATLAB SIMULINK and a process disturbed by step change in inlet flow rate is obtained. The obtained response which represents first order transfer function with zero dead time.

$G(s)=\frac{k_{p} e^{-\tau_{d}(s)}}{\tau s+1}$

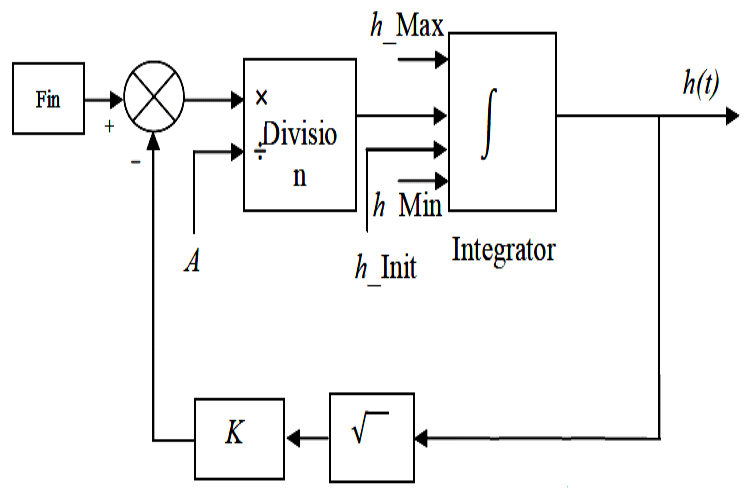

Figure 5: Mathematical Block Diagram of Tank System

Table 2. Model Parameters of Conical Tank

\begin{tabular}{|c|c|c|c|c|}
\hline \multirow[b]{2}{*}{ Region } & \multirow{2}{*}{$\begin{array}{l}\text { Inflow } \\
\text { rate } \\
\text { (LPH) }\end{array}$} & \multirow{2}{*}{$\begin{array}{c}\text { Height } \\
(\mathrm{cm})\end{array}$} & \multicolumn{2}{|c|}{ Direct Synthesis } \\
\hline & & & $\mathbf{K}_{\mathbf{P}}$ & $\mathbf{K}_{\mathbf{I}}$ \\
\hline 1 & $0-66$ & 1.44 & 1.88 & 24.39 \\
\hline 2 & $66-132$ & 5.76 & 7.339 & 4.166 \\
\hline 3 & 132-198 & 12.83 & 55.182 & 0.508 \\
\hline 4 & 198-264 & 23.04 & 305.194 & 0.0851 \\
\hline
\end{tabular}

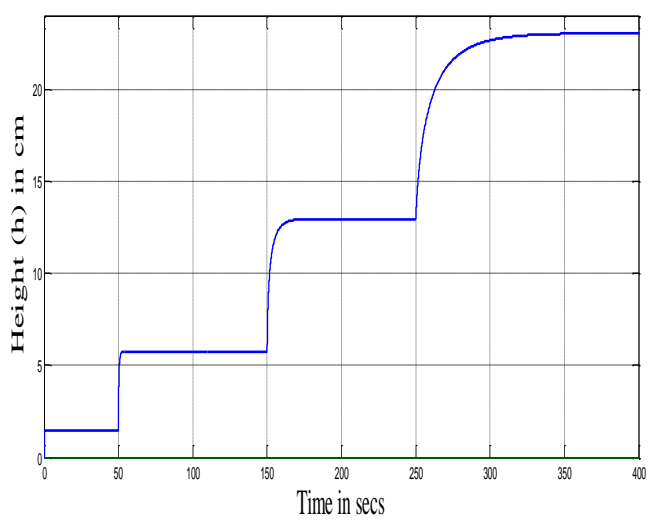

Figure 6: Simulated Response for height $(\mathrm{h})$ in $\mathrm{cm}$

Taking linearization in equation (9),

This is a nonlinear equation. To linearize this equation, Taylor's series method is utilized. That is, around $h=h s$,

$f(h)=(\sqrt{h})=\left(h^{\frac{1}{2}}\right) \cong\left(h^{\frac{1}{2}}\right)+\frac{1}{2}\left(h^{-\frac{1}{2}}(h-h s)+\right.$ higher order terms

$\frac{\partial \mathrm{h}}{\partial \mathrm{Fin}}=\left(\frac{\mathrm{R}}{\tau \mathrm{s}+1}\right)$

Where,

$$
C_{1}=\beta C ; C=\left(\frac{1}{2 \sqrt{h-h_{s}}}\right) ; \tau=A R ; R=\frac{1}{\beta C}
$$

The closed loop system transfer function described as,

$\frac{C(s)}{R(s)}=\left(\frac{R_{p}\left(T_{i} s+1\right)}{\left[(\tau s+1)\left(T_{i} s\right)\right]+\left[R_{p}\left(T_{i} s+1\right]\right.}\right)$

Where PI controller parameters were implemented to obtain the closed loop response with unity feedback.

Characteristics equation of the closed loop transfer function is derived as,

$\tau \mathrm{T}_{\mathrm{i}} \mathrm{S}^{2}+\mathrm{T}_{\mathrm{i}} \mathrm{S}+\mathrm{Rk}_{\mathrm{p}} \mathrm{T}_{\mathrm{i}} \mathrm{S}+\mathrm{Rk}_{\mathrm{p}}=0$

$\mathrm{S}^{2}+\left[\frac{1}{\tau}+\frac{\mathrm{Rk}_{\mathrm{p}}}{\tau}\right] \mathrm{S}+\frac{\mathrm{Rk}_{\mathrm{p}}}{\tau \mathrm{T}_{\mathrm{i}}}=0$

Splitting and rearranging the terms in equation (15),

$\mathrm{S}\left(\mathrm{S}+\frac{1}{\tau}\right)+\left(\frac{\mathrm{Rk}_{\mathrm{p}}}{\tau} \mathrm{S}+\frac{\mathrm{Rk}_{\mathrm{p}}}{\tau \mathrm{T}_{\mathrm{i}}}\right)=0$

If we assume,

$\frac{1}{\tau}=\frac{\mathrm{Rk}_{\mathrm{p}}}{\tau \mathrm{T}_{\mathrm{i}}}$ 
$\frac{\mathrm{Rk}_{\mathrm{p}}}{\tau}=1$

We get,

$(S+1)\left(\frac{R k_{p}}{\tau} S+\frac{R k_{p}}{\tau T_{i}}\right)=0$

From equation 17 and 18, PI parameter values were obtained,

$\mathrm{k}_{\mathrm{p}}=\frac{\tau}{\mathrm{R}}$

$\mathrm{T}_{\mathrm{i}}=\tau$

The above equations (20) and (21) which represents the relationship between tank system parameters and PI controller parameters. These two equations are used to implement gain scheduling controller.The parameter $\tau$ has scheduling variable, $\tau$ is replaced by $A R$, the parameter $A$ is change with respect to variation in level is acts as scheduling variable was calculated from the measurement of height(h). Using this A, R and $\tau$ controller parameters $K_{p}$ and $T_{i}$ were calculated and implemented in PI controller to make controller as gain scheduling controller. Simulation is performed using MATLAB and the simulated response which gives better response than Synthesis method.

\section{SIMULATION RESULTS}

The adaptive controller was designed using gain scheduling method and Figure 7 simulated response using adaptive controller. In this approach, the relation between tank parameters and PI controller parameters were derived and simulation results were obtained. Simulation result of Synthesis PI and with the various Operating point were obtained. The simulations were carried out in MATLAB Environment. The performance of the controller is compared on the basis of Rise Time, Settling Time and Over Shoot.

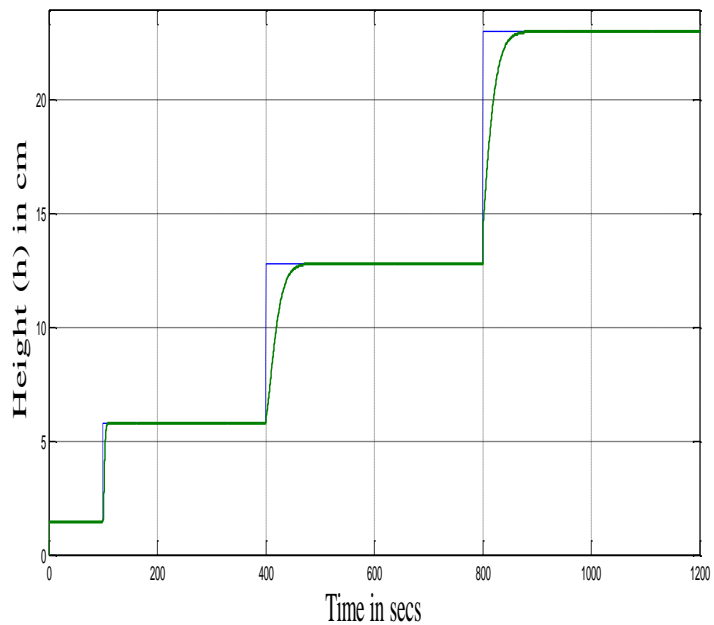

Figure 7: Simulation of height with respect setpoint change
Table 3. Comparison of synthesis PI and gain scheduling

\begin{tabular}{|l|l|l|l|}
\hline $\begin{array}{l}\text { Set Point } \\
(\mathbf{c m})\end{array}$ & Controller & $\begin{array}{l}\text { Settling time } \\
(\text { secs })\end{array}$ & \multicolumn{1}{|c|}{ ISE } \\
\hline 1.44 & Synthesis & 17.41 & 2.543 \\
& GS method & 1.741 & 0.4436 \\
\hline 5.76 & Synthesis & 37.3 & 71.52 \\
\cline { 2 - 4 } & GS method & 10.2 & 33.2 \\
\hline 12.83 & Synthesis & 342.3 & 703.2 \\
\cline { 2 - 4 } & GS method & 93.4 & 563 \\
\hline 23.04 & Synthesis & 4548 & 2143 \\
\cline { 2 - 4 } & & 152.3 & 754 \\
\cline { 2 - 4 } & GS method & & \\
\hline
\end{tabular}

\section{CONCLUSION}

An implementation of adaptive control by gain scheduling technique to a conical tank level system using MATLAB SIMULINK is performed. The performance of the adaptive control based controller is compared to direct synthesis method based PI controller tuning settings. The performance is compared for different set points like 1.44, 5.76, 12.83 and $23.04 \mathrm{~cm}$. For the conventional controller, it takes much time to reach the set point. The gain scheduling adaptive control based PI controller tracks the set point faster with minimum rise time and quick settling time response.

\section{REFERENCES}

[1] B Ziegler, G. and Nichols, N. B,.Optimum settings for automatic controllers, Trans. ASME, 64, 1942 , PP. 759768.

[2] Sundaresan K. R, Krishnaswamy R. R, Estimation of time delay, time constant parameters in Time, Frequency and Laplace Domains, Journal of Chemical Engineering., 56, 257, 1978.

[3] Rahul Shridhar, Douglas J.Cooper," A Novel Tuning For Multivariable MPC,"ISA Transactions,vol - 36,No.4,PP 273-280.

[4] S. J. Qin and G. Borders, A multi region fuzzy logic controller for nonlinear process control”, IEEE Trans. on Fuzzy Systems, 2, 1994 ,PP.74-81.

[5] Rahul Shridhar, Douglas J.Cooper," A Tuning Strategy for Unconstrained Multivariable MPC," Ind. Eng. Chern. Res. 1998, 37, 4003-4016.

[6] Hirotaka Yoshida, Kenichi Kawata, Yoshikazu Fukuyana, Yosuke Nakanishi, A particle swarm optimization for reactive power and voltage control considering voltage stability, IEEE international conference on intelligent system applications to power systems (ISAP'99), Rio de Janeiro, April 4-8 1999. 
[7] Parsopoulos and M. N. Vrahatis, Particle swarm optimizer in noisy and continuously changing environment, Indianapolis, IN, 2001.

[8] Swati Mohanty," Artificial neural network based system Identification and model predictive control of a flotation Column. Journal of Process Control 19 (2009),PP. 991999.

[9] N.S. Bhuvaneswari , G. Uma , T.R. Rangaswamy," Adaptive and optimal control of a non-linear process using Intelligent controllers," Applied Soft Computing 9, $182-190$

[10] S.Nithya, N.Sivakumaran, T.K.Radhakrishnan and N.Anantharaman" Soft Computing Based Controllers Implementation for Non-linear Process in Real Time" Proceedings of the World Congress on Engineering and Computer Science (WCECS )2010, Vol - 2 .
[11] V.R.Ravi, T.Thyagarajan, "Application of Adaptive Control Technique to Interacting Non Linear Systems "Electronics Computer Technology (ICECT), 2011 3rd International Conference on8-10 April 20112 PP: $386-$ 392.

[12] V.R.Ravi, T.Thyagarajan, M.Monika Darshini "A Multiple Model Adaptive Control Strategy for Model Predictive controller for Interacting Non Linear Systems "International Conference on Process Automation, Control and Computing (PACC),July 2011.PP:1 - 8.

[13] Sukanya R. Warier, SivanandamVenkatesh "Design of Controllers based on MPC for a Conical Tank System"IEEE-International Conference On Advances In Engineering, Science And Management (ICAESM 2012) March 30, 31, 2012 